\title{
ENSTROPHY DISSIPATION AND VORTEX THINNING FOR THE INCOMPRESSIBLE 2D NAVIER-STOKES EQUATIONS
}

\author{
IN-JEE JEONG AND TSUYOSHI YONEDA
}

\begin{abstract}
Vortex thinning is one of the main mechanisms of two-dimensional turbulence. By direct numerical simulation to the two-dimensional Navier-Stokes equations with small-scale forcing and large-scale damping, Xiao-Wan-Chen-Eyink (2009) found an evidence that inverse energy cascade may proceed with the vortex thinning mechanism. On the other hand, Alexakis-Doering (2006) calculated upper bound of the bulk averaged enstrophy dissipation rate of the steady-state two dimensional turbulence. In this paper, we show that vortex thinning induces enhanced dissipation with strictly slower vanishing order of the enstrophy dissipation than $R e^{-1}$.
\end{abstract}

\section{INTRODUCTION}

The mechanism of forward and inverse energy cascade of two-dimensional turbulence has been attracting many physicists but nevertheless is not well clarified so far. Theoretical studies of 2D turbulence usually employ statistics and sometimes impose assumptions on homogeneity isotropy. Alexakis and Doering [1] derived some rigorous upper bounds for the long time averaged bulk energy and enstrophy dissipation rates for $2 \mathrm{D}$ statistically stationary flows sustained by a variety of driving forces. In particular, they showed the enstrophy dissipation vanishes in the order $R e^{-1}$ when the external force is only on the single scale. They are emphasizing that, in this $R e^{-1}$ scaling, the flow exhibits laminar behavior, since energy is concentrated at relatively long length scales (independent of Reynolds number). Thus, their result tells us that, at least, 2D turbulence behavior must provide vanishing order which is strictly slower than the order $R e^{-1}$. Let us explain more precisely. The two dimensional Navier-Stokes equations are described as follows:

$$
\left\{\begin{array}{l}
\partial_{t} u-\nu \Delta u+u \cdot \nabla u=-\nabla p+f, \quad t \geq 0, x \in(\mathbb{R} / 2 \mathbb{Z})^{2} \\
\operatorname{div} u=0 \\
u(t=0)=u_{0}
\end{array}\right.
$$

where $u=u(t, x)=\left(u_{1}(t, x), u_{2}(t, x)\right), p=p(t, x)$ and $f=\left(f_{1}(t, x), f_{2}(t, x)\right)$ denote the velocity field, the pressure function of the fluid and the external force respectively. The case $\nu=0$ is called the Euler equations. Introducing the vorticity $\omega=\nabla \times u$, we can rewrite (1) as the following $2 \mathrm{D}$ vorticity equations:

$$
\partial_{t} \omega-\nu \Delta \omega+(u \cdot \nabla) \omega=\nabla \times f, \quad x \in \mathbb{T}^{2}:=(\mathbb{R} / 2 \mathbb{Z})^{2},
$$

where the velocity $u$ is determined by the (periodic) 2D Biot-Savart law:

$$
u(t, x)=\int_{\mathbb{T}^{2}} K_{2}(x-y) \omega(t, y) d y
$$

with

$$
K_{2}(x)=\frac{1}{2 \pi} \frac{\left(-x_{2}, x_{1}\right)}{|x|^{2}} \quad \text { (with reflections). }
$$

Date: June 11, 2020.

2010 Mathematics Subject Classification. Primary 35Q35; Secondary 35B30; Tertiary 76 F99.

Key words and phrases. Euler equations, Navier-Stokes equations, enstrophy dissipation, vortex thinning. 
Assume that the external force $f$ involves only a single length scale, namely, $-\Delta f \sim k_{f}^{2} f$. Assume further that the flow is a statistical steady state of body forced two-dimensional turbulence. Then the solution $u$ (depending on $\nu$ ) to the Navier-Stokes equations (1) satisfy

$$
\nu\left\langle|\nabla \omega|^{2}\right\rangle=\chi \leq \nu k_{f}^{4} U^{2},
$$

where $\chi$ is the enstrophy dissipation, $\langle\cdot\rangle$ is some averaging (in this paper we regard it as both space and time averages) and $U$ is the representative velocity. Note that, mathematically, if the enstrophy is finite, then this $\chi$ is always vanishing for $\nu \rightarrow 0$ (see [10, Proposition 2] for example).

The aim of this paper is to find a specific vorticity configuration which provides strictly slower vanishing order of the enstrophy dissipation than $R e^{-1}$, and in this case "vortex thinning" should be a strong candidate for it. To be more precise, Xiao-Wan-Chen-Eyink ([21]) investigated inverse energy cascade in steady-state two-dimensional turbulence by direct numerical simulation of the two-dimensional Navier-Stokes equations with small scale forcing and large scale damping. In their numerical work, they used an alternative equation (with a damping term), and found strong evidence that inverse energy cascade may proceed with vortex thinning mechanism. According to their evidence, there is a tensile turbulent stress in directions parallel to the isolines of smallscale vorticity. Thus the small-scale circular vortex will be stretched into elliptical shape, which is nothing more than "vortex thinning" phenomena.

In this paper we focus on a sequence of prescribed initial vorticity which provides vortex thinning behavior, and show that the vortex thinning behavior provides strictly slower vanishing order of the enstrophy dissipation than $R e^{-1}$. Now we formulate mathematically this enstrophy dissipation problem more precisely. Let us find a sequence of viscosity $\left\{\nu_{n}\right\}_{n}\left(\nu_{n} \rightarrow 0\right)$, a sequence of initial vorticity $\left\{\omega_{0, n}^{\nu_{n}}\right\}_{n} \subset H^{1}\left(\mathbb{T}^{2}\right) \cap C^{\infty}\left(\mathbb{T}^{2}\right)$ with $\left\|\omega_{0, n}^{\nu_{n}}\right\|_{H^{1}} \lesssim 1$ and a function $\varphi$ with $\varphi(\nu) \rightarrow 0(\nu \rightarrow 0)$, such that the corresponding solutions to the Navier-Stokes equations $\left\{\omega_{n}^{\nu_{n}}\right\}_{n}$ satisfy the following: for any $T>0$ fixed,

$$
\liminf _{n \rightarrow \infty} \varphi\left(\nu_{n}\right) \frac{1}{T} \int_{0}^{T} \int_{\mathbb{T}^{2}}\left|\nabla \omega_{n}^{\nu_{n}}(t, x)\right|^{2} d x d t>0 .
$$

Since we are interested in a finite-time result, we shall neglect the external force and take $R e=\nu^{-1}$.

Note that, in physics, $H^{1}$-norm of vorticity is called "palinstrophy" (see [15, Section 8] for example). The above formulation is the most strict one, since we are imposing uniformly boundedness $\left\|\omega_{0, n}^{\nu_{n}}\right\|_{H^{1}} \lesssim 1$ and taking arbitrary time $T>0$. These are necessary to avoid "trivial enstrophy dissipation": If we choose $\left\{\omega_{0, n}\right\}_{n}$ satisfying $\left\|\omega_{0, n}\right\|_{H^{1}} \rightarrow \infty$, and choose $T_{n}\left(T_{n} \rightarrow 0, n \rightarrow \infty\right)$ to be $\sup _{0<t<T_{n}} \nu_{n}\left\|\omega_{0, n}-\omega_{0, n}^{\nu_{n}}(t)\right\|_{H^{1}}^{2}<\epsilon$ (for sufficiently small $\epsilon>0$ ) with $\nu_{n} \sim\left\|\omega_{0, n}\right\|_{H^{1}}^{-2}$, then we trivially have (c.f. [10, Section 6])

$$
\lim _{n \rightarrow \infty} \nu_{n} \frac{1}{T_{n}} \int_{0}^{T_{n}} \int_{\mathbb{T}^{2}}\left|\nabla \omega_{n}^{\nu_{n}}(t, x)\right|^{2} d x d t \sim 1 .
$$

This is nothing more than trivial enstrophy dissipation we want to avoid.

We now construct our initial data. Given any smooth radial bump function $0 \leq \phi \leq 1$ with support in the ball $B(0,1 / 4)$ let

$$
\phi_{0}\left(x_{1}, x_{2}\right)=\sum_{\varepsilon_{1}, \varepsilon_{2}= \pm 1} \varepsilon_{1} \varepsilon_{2} \phi\left(x_{1}-\varepsilon_{1}, x_{2}-\varepsilon_{2}\right) .
$$

We assume further that $\phi=1$ in the ball $B(0,1 / 8)$. Clearly, the function $\phi_{0}$ is odd with respect to both $x_{1}$ and $x_{2}$. Moreover, given $\ell_{0} \geq 10$ (to be determined later independently of $n$; see the proof of Proposition 2 below), we take $\tilde{\omega}_{0}$ to be a smooth function which is odd-odd and satisfy $\tilde{\omega}_{0}=0$ 
on $[0,1]^{2} \backslash\left[2^{-\ell_{0}+2}, 1-2^{-\ell_{0}+2}\right]^{2}$ and $\tilde{\omega}_{0}=1$ on $\left[2^{-\ell_{0}+3}, 1-2^{-\ell_{0}+3}\right]^{2}$. Given some non-negative and decreasing sequence of reals $\left\{a_{\ell}\right\}_{\ell \geq 1}$, we define

$$
\omega_{0, n}(x)=\phi_{2 n}(x)+\tilde{\omega}_{0}(x)+\sum_{\ell=\ell_{0}}^{n} a_{\ell} \phi_{\ell}(x),
$$

where $\phi_{\ell}(x)=\phi_{0}\left(2^{\ell} x\right)$. Note that the supports of $\phi_{\ell}$ are disjoint. We remark that $\tilde{\omega}_{0}$ is necessary to guarantee (14). The term $\sum_{\ell} a_{\ell} \phi_{\ell}$ ("Bourgain-Li" bubbles after the work [5]) creates a large rate-of-strain tensor at the origin, which thins the smallest scale vortex blob $\phi_{2 n}$.

Remark 1. In the proof of our main result, we take

$$
a_{\ell}=\ell^{-1 / 2-\epsilon} \quad \text { for some small } 0<\epsilon<1 / 4 \text {. }
$$

With this choice, we see that $\left\|\omega_{0, n}\right\|_{H^{1}} \lesssim 1$ for all $n$.

We mention that the relation between the vortex thinning process and palinstrophy $\left(H^{1}\right.$-norm of the vorticity) has already been studied. In [2, Section 6.3] (see also [17]), Ayala-Protas found a initial vorticity of 2D Navier-Stokes equations (with very high Reynolds number) which attains maximum growth of palinstrophy, by using their own optimizing method. They figured out that the initial vorticity has odd (in both $x_{1}$ and $x_{2}$ ) type of symmetry with two different scales formation (which seems very similar to our initial vorticity setting). On the other hand, some of mathematicians have showed that there is an initial vorticity in $H^{1}$ such that the value of $\|\nabla \omega(t)\|_{L^{2}}$ (palinstrophy) to the 2D-Euler flow instantaneously blows up. More precisely, Bourgain-Li [5] and Elgindi-Jeong [8] constructed solutions to the 2D-Euler equations which exhibit norm inflation in $H^{1}$ (see also [18]). Now we state our main result.

Theorem 1 (Lower bound on the enstrophy dissipation). Let $\left\{\omega_{n}^{\nu}(t)\right\}_{\nu, n}$ be the unique solution of the $2 D$ Navier-Stokes equations with viscosity $\nu>0$ and initial data $\omega_{0, n}$ given in (5). Then, there exist a continuous function $\varphi(\nu)$ with $\varphi(\nu) \rightarrow 0$ as $\nu \rightarrow 0$ and a sequence of viscosity constants $\nu_{n}>0$ converging to zero, such that for any fixed $T>0$,

$$
\liminf _{n} \varphi\left(\nu_{n}\right) \frac{1}{T} \int_{0}^{T} \int_{\mathbb{T}^{2}}\left|\nabla \omega_{n}^{\nu_{n}}(t, x)\right|^{2} d x d t \gtrsim 1 .
$$

Remark 2. In the proof, one sees that the growth of $\left\|\nabla \omega_{n}^{\nu_{n}}\right\|_{L^{2}}$ comes from vortex thinning of the smallest scale bubble $\phi_{2 n}$ in (5). The function $\varphi$ can be chosen as $\varphi(\nu)=\left(\log \frac{1}{\nu}\right)^{-c_{0}}$ for some absolute constant $c_{0}>0$. Finally, note that the sequence of initial data in (5) is convergent weakly in $H^{1}$, and moreover, we can obtain the same conclusion for the following initial data:

$$
\omega_{0, n}(x)=\frac{\phi_{2 n}(x)}{\left(S_{n} \delta\right)^{\frac{c_{0}}{4}}}+\tilde{\omega}_{0}(x)+\sum_{\ell=\ell_{0}}^{n} a_{\ell} \phi_{\ell}(x),
$$

where $S_{n}, \delta$ and $c_{0}$ are determined in Proposition 2, In this case the smallest-scale vorticity $\frac{\phi_{2 n}(x)}{\left(S_{n} \delta\right)^{\frac{c_{0}}{4}}}$ vanishes for $n \rightarrow \infty$.

Remark 3. Using somewhat similar construction, the authors considered enhanced energy dissipation under the $2+\frac{1}{2}$-dimensional Navier-Stokes flow: small-scale horizontal vortex blob being stretched by large-scale, anti-parallel pairs of vertical vortex tubes [11. The zeroth law states that, in the limit of vanishing viscosity, the rate of kinetic energy dissipation for solutions to the 3D incompressible Navier-Stokes equations becomes nonzero. The authors proved a version of the zeroth law satisfying the above, which implies in particular enhanced dissipation.

Notation. We write $A \lesssim B$ if there is an absolute constant $c>0$ such that $A \leq c B$. Similarly, $A \approx B$ if $A \lesssim B$ and $A \gtrsim B$. 


\section{Proof of the Main Result}

Our result is a consequence of the "large Lagrangian deformation" for the 2D Euler equations, which was first established in a work of Bourgain and Li [5]. However, their idea works only for a short time and relies on a contradiction argument. Even worse, they were not able to obtain any quantitative growth rate. To overcome these difficulties, we employ an induction argument accompanied by a careful scaling control. For additional technical improvements achieved in this paper, see the remarks following the proof of Proposition 2.

Let $\eta_{n}=\left(\eta_{n, 1}, \eta_{n, 2}\right)$ be the associated Lagrangian flow from $\omega_{0, n}$, which is given by

$$
\partial_{t} \eta_{n}(t, x)=u_{n}\left(t, \eta_{n}(t, x)\right) \quad \text { with } \quad \eta_{n}(0, x)=x \in \mathbb{T}^{2},
$$

where $u_{n}$ is the corresponding velocity associated with $\omega_{0, n}$. The proof of our main result is based on the following two propositions. The first proposition makes precise what we mean by large Lagrangian deformation.

Proposition 2 (Creation of large Lagrangian deformation). Let $\left\{a_{k}\right\}_{k=1}^{\infty}$ be a bounded sequence of non-negative reals, and $\omega_{n}(t)$ be the solution to the $2 D$ Euler equation with initial data as in (5) with associated Lagrangian flow $\eta_{n}$. Set $S_{k}:=S_{k-1}+a_{k}$ for $k \geq 1$ with $S_{0}:=1$. Assume that $S_{k}$ is divergent with $k$. Then, for some absolute constant $c_{0}>0$, we have

$$
-\frac{\partial u_{n, 1}}{\partial x_{1}}(t, 0)=\frac{\partial u_{n, 2}}{\partial x_{2}}(t, 0) \gtrsim \frac{1}{t} \mathbf{1}_{\left\{t \gtrsim S_{n}^{-1}\right\}}
$$

and

$$
\left|\frac{\partial \eta_{n, 1}}{\partial x_{1}}(t, 0)\right|=\left|\frac{\partial \eta_{n, 2}}{\partial x_{2}}(t, 0)\right| \gtrsim\left(S_{n} t\right)^{c_{0}}
$$

for all $t \in[0, \delta)$ with some absolute constant $\delta>0$ and $n$ sufficiently large.

Proposition 3. Fix $a_{\ell}=\ell^{-\frac{1}{2}-\frac{1}{8}}$ and $S_{n}:=1+\sum_{\ell=1}^{n} a_{\ell}$. Let $\omega_{n}(t)$ be the solution of the 2D Euler equation with initial data $\omega_{0, n}$ defined in (5). Then we have

$$
\left\|\nabla \omega_{n}(t)\right\|_{L^{2}} \gtrsim\left(S_{n} t\right)^{\frac{c_{0}}{2}}
$$

for $t \in[0, \delta]$ with the same $\delta>0$ as in Proposition Q.

Remark 4. Although the above propositions are stated in possibly small time interval $[0, \delta]$, we can take $\delta>0$ arbitrarily large simply by rescaling the initial data. More precisely, we just choose $\omega_{0, n}$ to be sufficiently small in the $L^{\infty}$-norm (nevertheless $S_{n}$ is always divergent). In the following, we shall assume that the propositions are valid for $[0, T]$ with some $T>0$ given.

Given the above propositions, we complete the proof of the main theorem.

Proof of Theorem 1. Since initial data is always smooth, we note that for each $\nu>0$, the unique solution $\left\{\omega_{n}^{\nu}\right\}$ exists globally-in-time. From now on the multiplicative constants in the estimates will depend implicitly on $T>0$. From the maximum principle $\left\|\omega_{n}(t)\right\|_{L^{\infty}}=1$, we have

$$
\left\|\nabla u_{n}(t)\right\|_{L^{\infty}} \lesssim \log \left\|\omega_{0, n}\right\|_{C^{1}} e^{C t} \lesssim n
$$

on the time interval $[0, T]$. Note that $\left\|\omega_{0, k}^{S}\right\|_{C^{1}}$ can be always controlled by taking sufficiently large $k$ (depending on $n$ ). Then we have, from the classical estimate (see [4] for example)

$$
\left\|\omega_{n}(t)\right\|_{H^{s}} \lesssim\left\|\omega_{0, n}(t)\right\|_{H^{s}} e^{C(s) \int_{0}^{t}\left\|\nabla u_{n}(\tau)\right\|_{L} \infty d \tau}
$$

that

$$
\left\|\omega_{n}(t)\right\|_{H^{s}} \lesssim 2^{c(s) n}
$$


for some constant $c(s)>0$ depending only on $s>1$ for any $s$ and $t \in[0, T]$. We note that the Navier-Stokes solutions satisfy the same bounds:

$$
\left\|\omega_{n}^{\nu}(t)\right\|_{H^{s}} \lesssim 2^{c(s) n}
$$

with constant independent of $\nu>0$. This is because we still have the maximum principle $\left\|\omega_{n}^{\nu}(t)\right\|_{L^{\infty}} \leq 1$ for all $t \geq 0$ and the $H^{s}$ estimate holds a fortiori for the Navier-Stokes. Taking $s>3$ and by the Sobolev embedding, we obtain

$$
\left\|\nabla^{2} \omega_{n}(t)\right\|_{L^{\infty}}+\left\|\nabla^{2} \omega_{n}^{\nu}(t)\right\|_{L^{\infty}} \lesssim 2^{c n} \quad \text { for } \quad t \in[0, T] .
$$

This is elementary but is the key in the estimates below.

2.1. $L^{2}$ inviscid limit estimate on the velocity. We compare the $2 \mathrm{D}$ Euler and Navier-Stokes equations of the velocity:

$$
\begin{aligned}
& \partial_{t} u_{n}^{\nu}+u_{n}^{\nu} \cdot \nabla u_{n}^{\nu}+\nabla p_{n}^{\nu}=\nu \Delta u_{n}^{\nu}, \\
& \partial_{t} u_{n}+u_{n} \cdot \nabla u_{n}+\nabla p_{n}=0 .
\end{aligned}
$$

By

$$
\int\left(u^{\nu} \cdot \nabla\right)\left(u_{n}^{\nu}-u_{n}\right) \cdot\left(u_{n}^{\nu}-u_{n}\right)=0
$$

then we see that

$$
\frac{1}{2} \frac{d}{d t}\left\|u_{n}^{\nu}-u_{n}\right\|_{L^{2}}^{2}+\int\left(u_{n}-u_{n}^{\nu}\right) \cdot \nabla u_{n} \cdot\left(u_{n}^{\nu}-u_{n}\right)=\nu \int \Delta u_{n}^{\nu} \cdot\left(u_{n}^{\nu}-u_{n}\right) .
$$

We handle the right hand side as follows:

$$
-\nu \int\left|\nabla u_{n}^{\nu}\right|^{2}+\nu \int \nabla u_{n}^{\nu}: \nabla u_{n}
$$

Then, using the Cauchy-Schwarz inequality and Young's inequality, we have

$$
\frac{d}{d t}\left\|u_{n}^{\nu}-u_{n}\right\|_{L^{2}}^{2} \lesssim\left\|\nabla u_{n}\right\|_{L^{\infty}}\left\|u_{n}^{\nu}-u_{n}\right\|_{L^{2}}^{2}+\nu\left\|\nabla u_{n}\right\|_{L^{2}}^{2} \lesssim n\left\|u_{n}^{\nu}-u_{n}\right\|_{L^{2}}^{2}+\nu,
$$

which gives

$$
\left\|u_{n}^{\nu}-u_{n}\right\|_{L^{2}}^{2} \lesssim \nu 2^{c n}
$$

for $t \in[0, T]$.

2.2. $H^{1}$ inviscid limit estimate. This time, we consider the 2D Navier-Stokes solutions in the vorticity form and compare it with the corresponding Euler solutions:

$$
\begin{aligned}
& \partial_{t} \omega_{n}^{\nu}+u_{n}^{\nu} \cdot \nabla \omega_{n}^{\nu}=\nu \Delta \omega_{n}^{\nu}, \\
& \partial_{t} \omega_{n}+u_{n} \cdot \nabla \omega_{n}=0 .
\end{aligned}
$$

Then using previous bounds,

$$
\begin{aligned}
\frac{d}{d t}\left\|\omega_{n}-\omega_{n}^{\nu}\right\|_{L^{2}}^{2} & \lesssim\left\|\nabla \omega_{n}\right\|_{L^{\infty}}\left\|u_{n}-u_{n}^{\nu}\right\|_{L^{2}}\left\|\omega_{n}-\omega_{n}^{\nu}\right\|_{L^{2}}+\nu\left\|\nabla \omega_{n}\right\|_{L^{2}}^{2} \\
& \lesssim \nu 2^{c n}\left\|\omega_{n}-\omega_{n}^{\nu}\right\|_{L^{2}}+\nu 2^{c n} .
\end{aligned}
$$

Thus we have

$$
\left\|\nabla u_{n}-\nabla u_{n}^{\nu}\right\|_{L^{2}} \lesssim\left\|\omega_{n}-\omega_{n}^{\nu}\right\|_{L^{2}} \lesssim \nu 2^{c n}
$$


2.3. $H^{2}$ inviscid limit estimate. Let $\partial$ be a spatial derivative. Here, we consider the 2D NavierStokes solutions in the vorticity-gradient form and compare it with the corresponding Euler solutions:

$$
\begin{aligned}
& \partial_{t} \partial \omega_{n}^{\nu}+\left(\partial u_{n}^{\nu} \cdot \nabla\right) \omega_{n}^{\nu}+\left(u_{n}^{\nu} \cdot \nabla\right) \partial \omega_{n}^{\nu}=\nu \Delta \partial \omega_{n}^{\nu} \\
& \partial_{t} \partial \omega_{n}+\left(\partial u_{n} \cdot \nabla\right) \omega_{n}+\left(u_{n} \cdot \nabla\right) \partial \omega_{n}=0 .
\end{aligned}
$$

Again using previous bounds,

$$
\begin{aligned}
\frac{d}{d t}\left\|\partial\left(\omega_{n}-\omega_{n}^{\nu}\right)\right\|_{L^{2}}^{2} & \lesssim\left\|\nabla \partial \omega_{n}\right\|_{L^{\infty}}\left\|u_{n}-u_{n}^{\nu}\right\|_{L^{2}}\left\|\partial\left(\omega_{n}-\omega_{n}^{\nu}\right)\right\|_{L^{2}} \\
& +\left\|\nabla \omega_{n}\right\|_{L^{\infty}}\left\|\partial\left(u_{n}-u_{n}^{\nu}\right)\right\|_{L^{2}}\left\|\omega_{n}^{\nu}-\omega_{n}\right\|_{L^{2}}+\nu\left\|\omega_{n}\right\|_{\dot{H}^{3 / 2}}^{2} \\
& \lesssim \nu 2^{c n}\left\|\nabla\left(\omega_{n}-\omega_{n}^{\nu}\right)\right\|_{L^{2}}+\nu 2^{c n} .
\end{aligned}
$$

Here we choose $\nu \sim 2^{-c n}$, this guarantees that

$$
\left\|\nabla\left(\omega_{n}-\omega_{n}^{\nu}\right)\right\|_{L^{2}} \lesssim 1
$$

2.4. Completion of the proof. Recall (9) from Proposition 2:

$$
\left\|\nabla \omega_{n}(t)\right\|_{L^{2}} \gtrsim\left(S_{n} t\right)^{c_{0}}
$$

for $t \in[0, T]$. Then

$$
\frac{1}{T} \int_{0}^{T}\left\|\nabla \omega_{n}(t)\right\|_{L^{2}}^{2} d t \gtrsim_{T} S_{n}^{2 c_{0}}
$$

Then we finally have

$$
\begin{aligned}
& \varphi\left(\nu_{n}\right) \frac{1}{T} \int_{0}^{T}\left\|\nabla \omega_{n}^{\nu}(t)\right\|_{L^{2}}^{2} d t \\
& \gtrsim \varphi\left(\nu_{n}\right) \frac{1}{T} \int_{0}^{T}\left\|\nabla \omega_{n}(t)\right\|_{L^{2}}^{2} d t-\varphi\left(\nu_{n}\right) \sup _{0<t<T}\left\|\nabla\left(\omega_{n}^{\nu}(t)-\omega_{n}(t)\right)\right\|_{L^{2}}^{2} \\
& \gtrsim_{T} \varphi\left(\nu_{n}\right) S_{n}^{2 c_{0}}-\varphi\left(\nu_{n}\right) .
\end{aligned}
$$

Here we set $\varphi\left(\nu_{n}\right) \sim\left(-\log \left(\nu_{n}\right)\right)^{-c_{0}}$, then we have the desired estimate.

\section{Creation of Lagrangian deformation}

In this section, we give the proofs of two propositions.

Proof of Proposition 2. Recall that the 2D flow map associated with the initial vorticity $\omega_{0, n}$ is denoted by $\eta_{n}=\left(\eta_{n, 1}, \eta_{n, 2}\right)$ and it satisfies

$$
\partial_{t} \eta_{n}(t, x)=u_{n}\left(t, \eta_{n}(t, x)\right) \quad \text { with } \quad \eta_{n}(0, x)=x \in \mathbb{T}^{2},
$$

where $u_{n}$ is the corresponding velocity associated with $\omega_{0, n}$. Then we have the following representation

$$
D \eta_{n}:=\left(\begin{array}{cc}
\partial_{1} \eta_{n, 1} & \partial_{2} \eta_{n, 1} \\
\partial_{1} \eta_{n, 2} & \partial_{2} \eta_{n, 2}
\end{array}\right), \quad D \eta_{n}^{-1}=\left(\begin{array}{cc}
\partial_{2} \eta_{n, 2} & -\partial_{2} \eta_{n, 1} \\
-\partial_{1} \eta_{n, 2} & \partial_{1} \eta_{n, 1}
\end{array}\right)
$$

Note that we always have $\left\|D \eta_{n}(t)\right\|_{L^{\infty}}=\left\|D \eta_{n}^{-1}(t)\right\|_{L^{\infty}}$. To begin with, we shall fix some $n$ large and write $\eta=\eta_{n}$ for simplicity. Moreover, we note that $S_{n}$ is divergent in $n$. We systematically use the following "Key Lemma" due to Kiselev and Sverak [14] (a version on $\mathbb{T}^{2}$ has been established by [22]). The version presented below written in the polar coordinates is given in [9, Lemma 5.1]. 
Key Lemma. Assume the vorticity on $\mathbb{T}^{2}$ is bounded and odd with respect to both axis. Then $u=\nabla^{\perp} \Delta^{-1} \omega$ satisfies

$$
u(t, r, \theta)=\left(\begin{array}{c}
\cos \theta \\
-\sin \theta
\end{array}\right) r I(t, r)+r B(t, r, \theta)
$$

for $|r| \leq 1 / 2$, where

$$
I(t, r):=\frac{4}{\pi} \int_{0}^{\pi / 2} \int_{2 r}^{1} \frac{\sin \left(2 \theta^{\prime}\right)}{s} \omega\left(t, s, \theta^{\prime}\right) d s d \theta^{\prime}
$$

and

$$
\|B(t)\|_{L^{\infty}} \leq C\|\omega(t)\|_{L^{\infty}}
$$

for some absolute constant $C>0$.

For simplicity, we shall set $I(t):=I(t, 0)$ which is well-defined as the vorticities we consider is always vanishing in a small neighborhood of the origin. We now give a brief outline of the argument. The goal is to estimate the time integration of the "key integral" $I(t)$ for some time interval $\left[0, t^{*}\right]$, since then we deduce from the ODE

$$
\frac{d}{d t} \partial_{2} \eta_{n, 2}(t, 0)=\partial_{2} u_{n, 2}(t, 0) \partial_{2} \eta_{n, 2}(t, 0), \quad \partial_{2} \eta_{n, 2}(0,0)=1
$$

and

$$
\partial_{2} u_{n, 2}(t, 0) \geq I(t, 0)-C
$$

that (assuming $I(t, 0)>2 C$ )

$$
\left|\partial_{1} \eta_{n, 1}\left(t^{*}, 0\right)\right| \geq \exp \left(\frac{1}{2} \int_{0}^{t^{*}} I(t) d t\right) .
$$

The assumption

$$
I(t, 0)>2 C
$$

is easily guaranteed by taking $\ell_{0}$ large, since the contribution to $I$ from $\tilde{\omega}_{0}$ diverges as $\ell_{0} \rightarrow+\infty$ (see [14]). In turn, we may write

$$
I(t)=\sum_{k=1}^{n} I_{k}(t)+I_{2 n}(t)
$$

where

$$
I_{2 n}(t):=\frac{4}{\pi} \int_{0}^{\pi / 2} \int_{0}^{1 / 2} \frac{\sin (2 \theta)}{r} \phi_{2 n}\left(\eta^{-1}(t)\right)(r, \theta) d r d \theta
$$

and

$$
I_{k}(t):=\frac{4}{\pi} \int_{0}^{\pi / 2} \int_{0}^{1 / 2} \frac{\sin (2 \theta)}{r} a_{k} \phi_{k}\left(\eta^{-1}(t)\right)(r, \theta) d r d \theta
$$

are the contributions to $I(t)$ from the bubble initially located at the scales $2^{-2 n}$ and $2^{-k}$ respectively. Our strategy is to establish the following assertion inductively in $k$ : The "shape" of the $k$-th bubble essentially remains the same within the time scale $t_{k}:=c_{1} / S_{k}$ for some absolute constant $c_{1}>0$. Here what we mean by shape will be made precise later; for now we just mention that as a 
consequence, it follows that $I_{k}(t) \geq c I_{k}(0)$ for $t \in\left[0, t_{k}\right]$, where $c>0$ is a universal constant. Assuming for a moment that this statement holds, we obtain that

$$
\int_{0}^{t_{k}} I_{k}(t) d t \gtrsim t_{k} I_{k}(0) \gtrsim \frac{a_{k}}{S_{k}}
$$

Then we have (since $I_{2 n}(t)$ is non-negative, we just drop it off)

$$
\int_{0}^{t^{*}} I(t) d t \geq \sum_{k=1}^{n} \int_{0}^{\min \left\{t_{k}, t^{*}\right\}} I_{k}(t) d t \gtrsim \sum_{1 \leq k \leq n, c_{1}<t^{*} S_{k}} \frac{a_{k}}{S_{k}}
$$

owing to the non-negativity of each $I_{k}(t)$. We now observe that, by approximating the sum with a Riemann integral,

$$
\sum_{k=1}^{n} \frac{a_{k}}{S_{k}} \approx \log \left(S_{n}\right)
$$

and taking $k^{*}$ be the smallest number satisfying

$$
S_{k^{*}} t^{*}>c_{1}
$$

(which exists by taking $n$ larger if necessary, since $t^{*}>0$ and we are assuming that the sequence $S_{k^{*}}$ is divergent for $k^{*} \rightarrow \infty$ )

$$
\sum_{k=1}^{k^{*}} \frac{a_{k}}{S_{k}} \approx \log \left(S_{k^{*}}\right) \approx \log \left(\frac{c_{1}}{t^{*}}\right) .
$$

This gives (8) with some positive constant $c_{0}$. Similarly, we obtain that as long as $t \gtrsim S_{n}$,

$$
\partial_{2} u_{n, 2}(t, 0) \gtrsim t^{-1}
$$

which is simply (7). Hence it is sufficient to prove that

$$
I_{k}(t) \gtrsim I_{k}(0), \quad t \in\left[0, t_{k}\right]
$$

uniformly in $k$. Below we shall formulate and prove a claim which implies the above lower bound.

Step I: some preparations

We make some simple observations regarding the evolution of the bubbles. Recall from the definition of $\phi_{0}$ that restricted on to the positive quadrant, there exist "rectangles"

$$
\bar{R}_{0}=\left\{(r, \theta): \bar{r}_{1}<r<\bar{r}_{2}, \bar{\theta}_{1}<\theta<\bar{\theta}_{2}\right\}
$$

and

such that

$$
\underline{R}_{0}=\left\{(r, \theta): \underline{r}_{1}<r<\underline{r}_{2}, \underline{\theta}_{1}<\theta<\underline{\theta}_{2}\right\}
$$

$$
\phi_{0}=1 \text { on } \underline{R}_{0} \quad \text { and } \quad \phi_{0}=0 \text { outside of } \bar{R}_{0} .
$$

We may set

$$
\frac{1}{2}<\bar{r}_{1}<\underline{r}_{1}<\underline{r}_{2}<\bar{r}_{2}<2
$$

and

$$
\frac{\pi}{6}<\bar{\theta}_{1}<\underline{\theta}_{1}<\underline{\theta}_{2}<\bar{\theta}_{2}<\frac{\pi}{3} .
$$

Now by simple scaling, with the $2^{-k}$-scaled rectangles $\underline{R}_{k}$ and $\bar{R}_{k}$, we have

$$
\phi_{k}=1 \text { on } \underline{R}_{k} \quad \text { and } \quad \phi_{k}=0 \text { outside of } \bar{R}_{k},
$$


still restricted on the first quadrant (more precisely on $[0,1]^{2}$ ). This time, take an even smaller rectangle:

$$
\underline{R}_{0}^{*}=\left\{(r, \theta): \underline{r}_{1}^{*}<r<\underline{r}_{2}^{*}, \underline{\theta}_{1}^{*}<\theta<\underline{\theta}_{2}^{*}\right\} \subset \underline{R}_{0}
$$

where we may set

$$
\underline{r}_{1}^{*}=\frac{2 \underline{r}_{1}+\underline{r}_{2}}{3}, \quad \underline{r}_{2}^{*}=\frac{\underline{r}_{1}+2 \underline{r}_{2}}{3}
$$

and similarly

$$
\underline{\theta}_{1}^{*}=\frac{2 \underline{\theta}_{1}+\underline{\theta}_{2}}{3}, \quad \underline{\theta}_{2}^{*}=\frac{\underline{\theta}_{1}+2 \underline{\theta}_{2}}{3} .
$$

Then as before define

$$
\underline{R}_{k}^{*}:=\left\{(r, \theta): \underline{r}_{1}^{*}<2^{k} r<\underline{r}_{2}^{*}, \underline{\theta}_{1}^{*}<\theta<\underline{\theta}_{2}^{*}\right\}
$$

Moreover, define

$$
\bar{A}_{k}:=\left\{(r, \theta): 2^{-k-1}<r<2^{1-k}, 0<\theta<\frac{\pi}{2}\right\} .
$$

We shall now prove the following

Claim. In the time interval $\left[0, t_{k}\right]$, the $k$-th bubble remains $a_{k}$ on the rectangle $\underline{R}_{k}^{*}$ and vanishes outside $\bar{A}_{k}$. Here $t_{k}:=c_{1} / S_{k}$ with $c_{1}>0$ independent of $k$.

This is what we mean by retaining the same "shape". We now rewrite the evolution of the trajectories in polar coordinates, using (12). Given some $x \in[0,1]^{2}$, we shall express the point $\eta(t, x)$ using $|\eta|$ and $\theta(\eta)$. Then,

$$
\begin{aligned}
\frac{d}{d t}|\eta| & =u(t, \eta) \cdot\left(\begin{array}{c}
\cos (\theta(\eta)) \\
\sin (\theta(\eta))
\end{array}\right) \\
& =|\eta|\left(\cos (2 \theta(\eta)) I(t,|\eta|)+\left(\cos (\theta(\eta)) B_{1}+\sin (\theta(\eta)) B_{2}\right)\right)
\end{aligned}
$$

and

$$
\begin{aligned}
\frac{d}{d t} \theta(\eta) & =\frac{u(t, \eta)}{|\eta|} \cdot\left(\begin{array}{c}
-\sin (\theta(\eta)) \\
\cos (\theta(\eta))
\end{array}\right) \\
& =-\sin (2 \theta(\eta)) I(t,|\eta|)+\left(-\sin (\theta(\eta)) B_{1}+\cos (\theta(\eta)) B_{2}\right)
\end{aligned}
$$

where $B=\left(B_{1}, B_{2}\right)$ is from (12) .

Step II: induction base case $k=1$

To proceed, we recall a simple estimate of Yudovich (see e.g. [8] for a proof):

Lemma 4. Let $\omega(t) \in L^{\infty}\left([0, \infty): L^{\infty}\left(\mathbb{T}^{2}\right)\right)$ be a solution of the $2 D$ Euler equations on $\mathbb{T}^{2}$, and $\eta$ be the associated flow map. Then for some absolute constant $c>0$, we have

$$
\left|x-x^{\prime}\right|^{1+c t \mid\left\|\omega_{0}\right\|_{L^{\infty}}} \leq\left|\eta(t, x)-\eta\left(t, x^{\prime}\right)\right| \leq\left|x-x^{\prime}\right|^{1-c t \mid\left\|\omega_{0}\right\|_{L^{\infty}}},
$$

for all $0 \leq t \leq 1$ and $\left|x-x^{\prime}\right| \leq 1 / 2$.

We shall be concerned with the bubble $\phi_{1}$ and the trajectories $\eta(t, x)$ where $x \in \operatorname{supp}\left(\phi_{1}\right)$. Using the Yudovich estimate (17) with $x^{\prime}=0$, we see that such trajectories are trapped inside the region $\left\{2^{-2} \leq r\right\}$ during $\left[0, t_{1}\right]$ by choosing $c_{1}>0$ depending only on $c\left\|\omega_{0}\right\|_{L^{\infty}}$ in (17). Similarly, trajectories starting from $\cup_{k>1} \operatorname{supp}\left(\phi_{k}\right)$ cannot cross the circle $\left\{r=2^{-2}\right\}$. This results in a naive bound

$$
I_{1}(t,|\eta|) \leq I_{1}\left(t, 2^{-2}\right) \lesssim a_{1} 2^{-2}\left\|\phi_{1}\left(\eta^{-1}(\cdot)\right)\right\|_{L^{1}} \lesssim a_{1}
$$


on the same time interval, where

$$
I_{k}(t, r):=\frac{4}{\pi} \int_{0}^{\pi / 2} \int_{2 r}^{1 / 2} \frac{\sin (2 \theta)}{s} a_{k} \phi_{k}\left(\eta^{-1}(t)\right)(s, \theta) d s d \theta .
$$

We use this to obtain slightly improved estimates on $|\eta|$ in (15):

$$
\left|\frac{d}{d t} \ln \frac{1}{|\eta|}\right| \lesssim S_{1}
$$

using $|B| \lesssim 1$. This guarantees that, given any small $\epsilon>0$, by taking $c_{1}=c_{1}(\epsilon)>0$ small enough if necessary, we have

$$
\left|\ln \frac{|\eta(0, x)|}{|\eta(t, x)|}\right|<\epsilon, \quad t \in\left[0, t_{1}\right],
$$

recalling that $t_{1}=c_{1} / S_{1}$. For the angle, we simply use

$$
\left|\frac{d}{d t} \theta(\eta)\right| \lesssim S_{1}
$$

to deduce

$$
|\theta(\eta(t, x))-\theta(\eta(0, x))|<\epsilon
$$

again for $t \in\left[0, t_{1}\right]$ by taking $c_{1}>0$ smaller if necessary. Thus, a suitable choice of $\epsilon>0$ (depending only on $\left.\underline{r}_{1}, \underline{r}_{2}, \underline{\theta}_{1}, \underline{\theta}_{2}\right)$ finishes the proof of the Claim for the case $k=1$.

Step III: completing the induction

We now assume that for some $k_{0}>1$ the Claim has been proved for $k=1, \cdots, k_{0}-1$. We are now concerned with the trajectories $\eta(t, x)$ where $t \leq t_{k_{0}}$ and $x \in \operatorname{supp}\left(\phi_{k_{0}}\right)$. The induction hypothesis guarantees that, as long as $2^{-\left(k_{0}+1\right)}<|\eta|<2^{-\left(k_{0}-1\right)}$, we have that

$$
\left|\frac{d}{d t} \ln \frac{1}{|\eta|}\right| \lesssim S_{k_{0}}
$$

simply because $t_{k}$ is decreasing with $k$ and the hypothesis ensures that the contribution of $a_{k} \phi_{k} \circ$ $\eta^{-1}(t)$ to the key integral (18) is bounded by $c a_{k}$ with some $c$ independent of $k$, for $k=1, \cdots, k_{0}-1$. More precisely

$$
I_{k}(t,|\eta|) \lesssim a_{k} 2^{-2 k}\left\|\phi_{k}\left(\eta^{-1}(\cdot)\right)\right\|_{L^{1}} \lesssim a_{k} .
$$

Strictly saying, here, we use the even smaller rectangles $\underline{R}_{k}^{*}$. Thus $c$ is depending on $\epsilon>0$. This implies that

$$
\left|\ln \frac{|\eta(0, x)|}{|\eta(t, x)|}\right|<\epsilon, \quad t \in\left[0, t_{k_{0}}\right]
$$

for the same $\epsilon$ and $c_{1}$. Similarly, we can deduce

$$
|\theta(\eta(t, x))-\theta(\eta(0, x))|<\epsilon
$$

on $\left[0, t_{k_{0}}\right]$. The proof of Claim is complete, which finishes the proof.

Remark 5. A few remarks are in order.

- Large Lagrangian deformation occurs at the origin. Proposition 2 shows that for bubbles satisfying $S_{n} \rightarrow \infty$ as $n \rightarrow \infty$, large Lagrangian deformation must occur, and it occurs even within a time interval that shrinks to zero for $n$ large. We emphasize that we can pinpoint the location of large Lagrangian deformation to be the origin (which was an open problem to the best of our knowledge), while using contradiction arguments it is possible 
(see [5, 8]), with less work, to show existence of large Lagrangian deformation (somewhere in the domain).

- Dichotomy for bubbles. Note that in the case when the sequence $a_{k}$ is summable, the initial vorticity belongs to the critical Besov space $B_{\infty, 1}^{0}$ uniformly in $n$ (for the rigorous calculation, see [18] for example). There is uniqueness and existence in this space $B_{\infty, 1}^{0}$ ([20]), which in particular guarantees that the corresponding velocity gradient is uniformly bounded in $n$ for a short time interval. Therefore, we have the following dichotomy for bubbles: short-time large Lagrangian deformation occurs if and only if the sequence $\left\{a_{k}\right\}$ is not summable.

- Sharpness of the growth rate. It can be shown that with the data in (5), we have

$$
|D \eta(t, 0)| \leq C\left(c_{2}\right), \quad t \in\left[0, c_{2} / S_{n}\right]
$$

for any fixed constant $c_{2}>0$. This follows from the well-posedness of the Euler equations with vorticity in $B_{\infty, 1}^{0}([19])$ and the fact that $\left\|\omega_{0, n}\right\|_{B_{\infty, 1}^{0}} \sim S_{n}$. Comparing this with (8), one sees that the lower bound is sharp at least during this time scale. Hence we must wait a bit longer to see large deformation at the origin.

- Case of the continuum. Our considerations equally apply well to the "continuum" version of the bubbles; that is, we may take locally

$$
\omega_{0, n}(r, \theta)=\varphi_{2^{-n-1}} *(g(r) \chi(\theta)), \quad 0 \leq r<1 / 2
$$

where $\chi \geq 0$ on $\theta \in[0, \pi / 2]$ and $\chi(\theta)=-\chi(-\theta)=-\chi(\pi-\theta)$, and $g \geq 0$ is a bounded continuous function on $[0,1 / 2] \rightarrow[0,1]$. Here $a_{k}$ corresponds to $g\left(2^{-k}\right)$ and $S_{k}$ to $\int_{2^{-k}}^{1} g(r) r^{-1} d r$. For an example, in the case $g(r)=|\ln r|^{-1 / 2-\epsilon}, \omega_{0}=g(r) \sin (2 \theta)$ belongs to $H^{1}$ (considered explicitly in [8]), and using the method in this paper one can show that the corresponding solution escapes $H^{1}$ without appealing to a contradiction argument.

Proof of Proposition 3. Recall that Proposition 2 established creation of large Lagrangian deformation at the origin for the solution of $2 \mathrm{D}$ Euler with initial data $\omega_{0, n}$. We now prove that the deformation persists on the support of $\phi_{2 n}$ and use this observation to complete the proof of Proposition 3 .

For convenience, we set $\omega_{0, n}^{S}=\phi_{2 n}$ and $\omega_{n}^{S}(t):=\omega_{0, n}^{S} \circ \eta_{n}^{-1}(t)$. We observe that for any $t$, $\left\|\omega_{n}(t)\right\|_{H^{1}} \geq\left\|\omega_{n}^{S}(t)\right\|_{H^{1}}$. From the definition, $\left\|\omega_{0, n}^{S}\right\|_{H^{1}} \gtrsim 1$. Moreover, we note that for $t \in[0, \delta]$, the support of $\omega_{n}^{S}(t)$ is contained in the ball of radius $2^{-n}$ centered at the origin. This can be easily shown using Lemma 4 (we choose $\delta=\frac{1}{2 c|| \omega_{0} \|_{L^{\infty}}}$ ). Recalling the simple estimate

$$
\left\|\nabla u_{n}(t)\right\|_{C^{\frac{1}{2}}} \lesssim\left\|\omega_{n}(t)\right\|_{C^{\frac{1}{2}}} \lesssim 2^{\frac{n}{2}},
$$

we have

$$
\left|\nabla u_{n}(t, x)-\nabla u_{n}(t, 0)\right| \lesssim 2^{\frac{n}{2}}|x|^{\frac{1}{2}}
$$

so that for $x \in \operatorname{supp}\left(\omega_{n}^{S}(t)\right)$,

$$
\left|\nabla u_{n}(t, x)-\nabla u_{n}(t, 0)\right| \lesssim 1
$$

In particular, since $\partial_{1} u_{n, 2}(t, 0)=\partial_{2} u_{n, 1}(t, 0)=0$ by the odd symmetry,

$$
\left|\partial_{1} u_{n, 2}(t, x)\right|+\left|\partial_{2} u_{n, 1}(t, x)\right| \lesssim 1 \text {. }
$$

We now fix some $x \in \operatorname{supp}\left(\omega_{0, n}^{S}\right)$, and estimate (dropping the subscript $n$ for simplicity)

$$
\frac{d}{d t} \nabla \eta(t, x)=\nabla u(t, 0) \nabla \eta(t, x)+(\nabla u(t, \eta)-\nabla u(t, 0)) \nabla \eta(t, x) .
$$


This can be estimated as follows:

$$
\frac{d}{d t}|\nabla \eta(t, x)| \geq(|\nabla u(t, 0)|-C)|\nabla \eta(t, x)| .
$$

Since $\nabla \eta(t, x)=$ Id at $t=0$, for sufficiently large $n$, one can show easily from the lower bound of $\partial_{1} u_{1}(t, 0)$ that

$$
\left|\partial_{1} \eta_{n, 1}(t, x)\right|=\left|\partial_{2} \eta_{n, 2}(t, x)\right| \gtrsim\left(S_{n} t\right)^{\frac{c_{0}}{2}}
$$

Finally,

$$
\nabla \omega_{n}^{S}(t)=\nabla \omega_{0, n}^{S} \circ \eta_{n}^{-1} \nabla \eta_{n}^{-1}
$$

and recalling the representation formula for $\nabla \eta_{n}^{-1}$ in terms of $\nabla \eta_{n}$ (see (11) ) and $\left\|\nabla \omega_{0, n}^{S}\right\|_{L^{2}} \gtrsim 1$, we obtain

$$
\left\|\omega_{n}(t)\right\|_{H^{1}} \geq\left\|\nabla \omega_{n}^{S}(t)\right\|_{L^{2}} \gtrsim \inf _{x \in \operatorname{supp}\left(\omega_{0, n}^{S}\right)}\left|\partial_{1} \eta_{n, 1}\right| \gtrsim\left(S_{n} t\right)^{\frac{c_{0}}{2}}
$$

which finishes the proof.

\section{Conclusion}

We prepared small-scale vortex blob and multi-scale odd-symmetric vortices for the initial data, and showed that the corresponding 2D Euler flow creates vortex thinning. In turn, using this thinning, we showed that the corresponding 2D Navier-Stokes flow induces the enstrophy dissipation with strictly slower decaying rate than $R e^{-1}$.

Acknowledgments. We thank Professor A. Mazzucato for inspiring communications and telling us about the article [10]. Research of TY was partially supported by Grant-in-Aid for Young Scientists A (17H04825), Grant-in-Aid for Scientific Research B (15H03621, 17H02860, 18H01136, 18H01135 and 20H01819), Japan Society for the Promotion of Science (JSPS). IJ has been supported by the POSCO Science Fellowship of POSCO TJ Park Foundation and the National Research Foundation of Korea (NRF) grant (No. 2019R1F1A1058486).

\section{REFERENCES}

1. A. Alexakis and C. R. Doering, Energy and enstrophy dissipation in steady state 2d turbulence, Phys. Letters A 359, (2006) 652-657.

2. D. Ayala and B. Protas, Maximum palinstrophy growth in 2D incompressible flows, J. Fluid Mech. 742 (2014), 340367.

3. H. Bahouri and J.-Y. Chemin, Équations de transport relatives á des champs de vecteurs non-lipschitziens et mécanique des fluides, Arch. Rational Mech. Anal., 127 (1994), 159-181.

4. J. T. Beale, T. Kato and A. Majda, Remarks on the breakdown of smooth solutions for the 3-D Euler equations, Commun. Math. Phys., 94 (1984), 61-66.

5. J. Bourgain and D. Li, Strong ill-posedness of the incompressible Euler equations in borderline Sobolev spaces, Invent. math. 201, (2015), 97-157; preprint arXiv:1307.7090 [math.AP].

6. J. Chemin, Perfect Incompressible Fluids, Clarendon Press, Oxford 1998.

7. P. Constantin, The Littlewood-Paley Spectrum in Two-Dimensional Turbulence, Theo. Comput. Fluid Dynam., 9, (1997), 183-189.

8. T. Elgindi and I.-J. Jeong, Ill-posedness for the incompressible Euler equations in critical Sobolev spaces, Ann. PDE, 3 (2017), 7.

9. T. Elgindi and I.-J. Jeong, On Singular Vortex Patches, I: Well-posedness Issues, arXiv:1903.00833.

10. M. C. L. Filho, A. L. Mazzucato and H. J. N. Lopes, Weak solutions, renormalized solutions and enstrophy defects in $2 D$ turbulence, Arch. Ration. Mech. Anal., 179 (2006), 353387.

11. I.-J. Jeong and T. Yoneda, Vortex stretching and enhanced dissipation for the incompressible 3D Navier-Stokes equations, Math. Annal., to appear, arXiv:2001.02333 
12. T. Kato, On classical solutions of the two-dimensional non-stationary Euler equation, Arch. Ration. Mech. Anal. 25 (1967), 188-200.

13. T. Kato, Remarks on the Euler and Navier-Stokes equations in $\mathbb{R}^{2}$, Proc. Sym. Pure Math., 45 (1986), 1-7.

14. A. Kiselev and V. Šverák, Small scale creation for solutions of the incompressible two-dimensional Euler equation, Ann. of Math., 180 (2014) 1205-1220.

15. M. Lesieur, Turbulence in Fluids (Fluid Mechanics and Its Applications), Springer, the Netherlands, 2007.

16. A. Majda and A. Bertozzi, Vorticity and Incompressible Flow, Cambridge University Press, Cambridge 2002.

17. W. H. Matthaeus, W. T. Stribling, D. Martinez, S. Oughton and David Montgomery, Selective decay and coherent vortices in two-dimensional incompressible turbulence, Phys. Rev. Lett. 66 (1991), 2731-2734.

18. G. Misiołek and T. Yoneda, Continuity of the solution map of the Euler equations in Hölder spaces and weak norm inflation in Besov spaces, Trans. Amer. Math. Soc., 370 (2018), 4709-4730.

19. H. Pak and Y. Park, Existence of solution for the Euler equations in a critical Besov space $B_{\infty, 1}^{1}\left(\mathbb{R}^{n}\right)$, Comm. Partial Differential Equations 29 (2004), no. 7-8, 11491166.

20. M. Vishik, Hydrodynamics in Besov spaces, Arch. Rational Mech. Anal. 145 (1998), 197-214.

21. Z. Xiao, M. Wan, S. Chen and G. L. Eyink, Physical mechanism of the inverse energy cascade of two-dimensional turbulence: a numerical investigation, J. Fluid Mech. 619 (2009), 1-44.

22. A. Zlatoš, Exponential growth of the vorticity gradient for the Euler equation on the torus, Adv. Math., $\mathbf{2 6 8}$ (2015), 396-403

School of Mathematics, Korea Institute for Advanced Study, Seoul, Republic of Korea

E-mail address: ijeong@kias.re.kr

Graduate School of Mathematical Sciences, University of Tokyo, Komaba 3-8-1 Meguro, Tokyo 153-8914, JAPAN

E-mail address: yoneda@ms.u-tokyo.ac.jp 